\title{
On the Aesthetic Characteristics of Chinese Characters in Art Design
}

\author{
Sun Hongyang ${ }^{1}$ and Hong Changseok ${ }^{2}$ \\ (1 East China University of Technology, Nanchang 330013, China, 398188424@qq.com) \\ (2 Chonbuk National University, Quanzhou 561756,Korea)
}

Keywords: Chinese character; Art design; Aesthetic characteristics

\begin{abstract}
Objective: To study the characteristics of aesthetic Chinese characters in art design. Methods: Through the analysis of the Chinese characters generation, development and mature process, and explained from the aesthetic features and design Chinese characters itself perspective. At the same time the combination of traditional Chinese culture, the theory of art, with works of art and design of these features are confirmed. Conclusion: In art design, Chinese characters Inclusions the image and the beauty of structure, art and the beauty of rhyme, design and emotional beauty.
\end{abstract}

\section{Introduction}

Although the basic strokes of Chinese characters only have eight kinds of "points, horizontal, vertical, apostrophes, bending, bending and hooks", the cultural connotation and aesthetic characteristics of the Chinese characters are impressive and profound. Triggered by Chinese characters, such as various forms of art also flourished, such as calligraphy, seal cutting, folk art words and Chinese characters as main elements of poster design, packaging design of visual communication design to reflect the characters of its excellent brilliant light, and form a strong national style. Today, Chinese characters is not only a record of life and expressing the culture a tool, but also a highly aesthetic value of plastic arts, in the practice of the art design occupying an increasingly important position.

\section{The Iconicity and Structural Beauty of Chinese Characters}

\section{Iconicity}

"Chinese characters are the most vital signs, which are born out of hieroglyphics, and the spirit of the text is reflected in abstract strokes, and the spirit of the world is in harmony with the spirit of heaven and earth, with the function of form and ideality" [1]. The "hieroglyphs" method of "hieroglyphics" in the Chinese characters is the iconicity of Chinese characters. The pictograph refers to the physical appearance of the object, or the physical appearance of the object, or the shape of the figure. For example, the "moon" in Jinmen "moon" is like a curved one. "mountain" is like a rolling mountain. "water" is like a continuous spring. "fish" is like a fish with a tail, as shown in Fig.1.
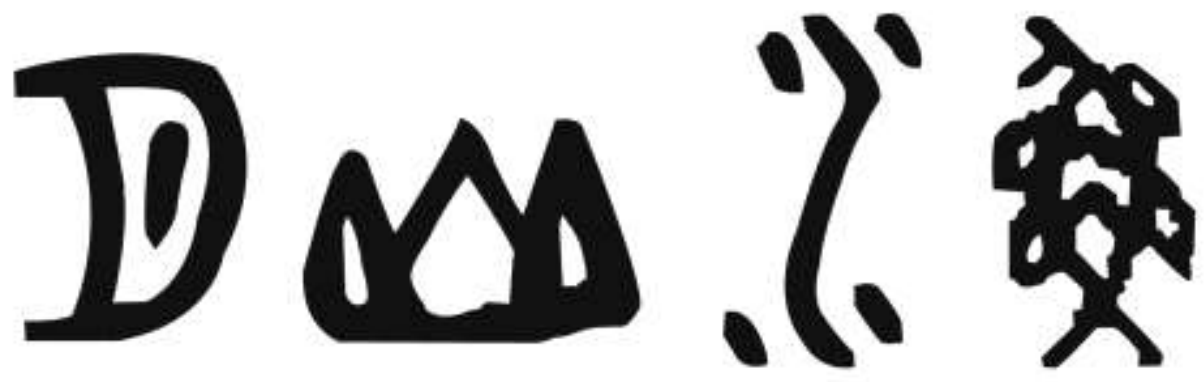

Figure 1. Bronze inscriptions: "month", "mountain" and "water", "fish"

Calligraphy and painting homology, but Chinese characters vivid simulations is not only to simple 
object, but rather "nearly in the body, far from the" [2], is a blend of people's life experience and subjective spirit of abstraction and generalization. It is more concise and refined than pure painting, has a strong symbolic color, and has the visual communication meaning needed for artistic design. For example, the oracle "ox", in the symbol of the arc of the horn, added a horizontal line, forming a visual symbol "like and not like", as shown in Fig.2.At the same time, Chinese characters with fluent line, bold hand in photograph reflect with strong forms of brushwork of object inductive generalization and abstraction refinement process itself is a kind of art design and visual communication to create.

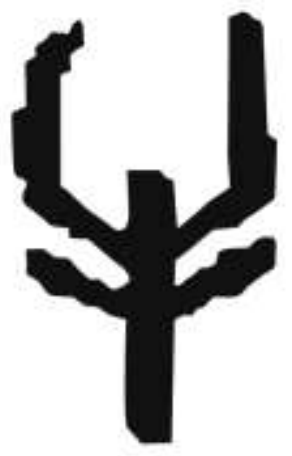

Figure 2. Oracle-bone: "cattle"

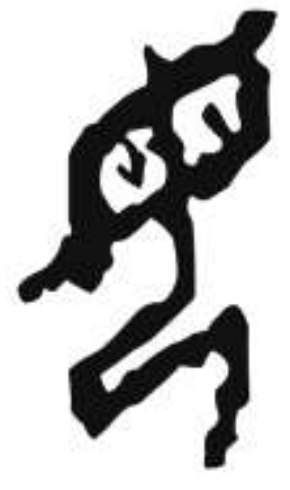

Figure 3. Oracle-bone: "mother"

\section{The Beauty of the Structure}

The structural beauty of Chinese characters is mainly embodied in the beauty of the dots and lines. Each point, each picture is vivid and fluent in the image, the structure is harmonious and full of verve. Such as oracle "mother" for the two points on the chest of the "lady" is said a woman's breasts, and suggest that reproductive image of lactation, the spot, the line is exquisite, modelling is generated, which fully reflects the structure features of the calligraphy and painting homology, as shown in Fig.3. Points, lines with Chinese characters and the basic elements of art design ideas and elicits emotions, characters of dot, line extremely rich aesthetic feeling, rich change, straight, curved, long and short, like, Athens and the beauty of simplicity.

With the richness of writing tools and materials, the basic forms of Chinese characters and lines are increasing, and the decorative and aesthetic features are increasingly diversified. People's attention to the change of Chinese character points and lines has been greatly enhanced, making Chinese characters an independent art--Calligraphy. Jin Dynasty calligrapher WeiFuRen brilliantly: in "BiZhen Figure" calligraphy "Dot", such as rock fall, "Peak" and "horizontal" such as "li array cloud", "vertical" such as "long live the withered cane", "left", such as "land is broken horn", "Si", such as "Collapse wave LeiBen", "Discount" such as "A small mind JinJie a tidy mind", "Tick" such as "Hundreds of jun if"; Think that calligraphy pen seal character to "Circle structure prepared", Zhang Cao to "on" and "dangerous and terrible," malapropism book BaFenShu want to "gentle and graceful discrepancy, the official script" to "depression", if can "for each word, each as its shape", the "Si miao yi, saying until tomorrow."[3] it can be seen that calligraphy shows people's understanding and expression of Chinese character points and lines, and points and lines are the soul and bones of Chinese characters. Hand made by heart, point, line length, width, size, height, heavy, pitch, light and inclusive, show embellish, acute disease, the density, the Angle is, let and among primary and secondary, are all a hotchpotch of inner feelings are like traces. Chinese characters in the beginning was to pursue a kind of image and the structure of the aesthetic feeling, or XiongJin of primitive simplicity, or strength forms beautiful, flowing, or romantic and bold and unrestrained, all showing a harmonious beauty. As Zong Baihua said: "the Chinese pen, beginning with a painting, has broken the void, leaving the handwriting, both the beauty of the human heart and the beauty of all things. "[4] 


\section{The Application of Iconicity and Structural Beauty in Design}

The image and structure of Chinese characters are more and more concerned by designers. In design, Chinese character table shape and feeling the dual characteristics of the complex information of concise and streamline, at the same time will be vivid and structural beauty show incisively and vividly, make the work more cultural connotation and visual impact, showing strong ethnic characteristics and aesthetic taste. "The characteristics of the image composition design is to grasp the specific text personalized image character, the connotation of the literal qualities through visual expression form its own interest, through the inner meaning and external form clearly show the influence of" [5]. As the symbol of the people's bank of China designed by Chen Hanmin, the "man" glyph is an element with distinct allegorical and figurative characteristics, as shown in Fig.4. And like a "family on both sides of the strait" poster of Yu Bingnan, in a different font image of "home" word, the main body of a white cursive "home" word, lower right changes for the Taiwan topography, vividly convey the mainland, Taiwan, on both sides of a concept, visual intense and rich cultural connotation, as shown in Fig.5.



Figure 4. The China People's Bank logo

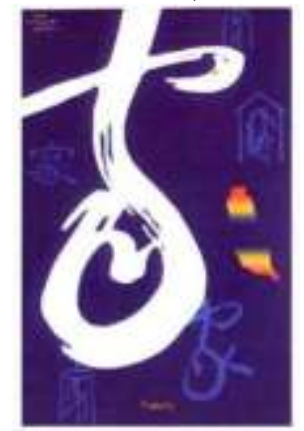

Figure 5. Cross-strait one family

\section{The Artistic and Rhythmic Beauty of Chinese Characters}

\section{Artistic}

The artistic quality of Chinese characters through the change of the pen and ink, by speed, structure, style, emotional expression of the author in the works and has a unique shape and national aesthetic concept, aesthetic value and significance of independence. As aesthetes li Zehou said: "the Chinese characters form in obtained independent of symbol meaning (meaning), with its purified the line freedom than the abstract geometric lines of painted pottery decorative design in a utensil and the matters and more diverse lines of movement and spatial structure, showing a variety of body posture, emotional off and imposing manner, finally formed the Chinese unique line art, calligraphy." [6] is calligraphy calligrapher's wisdom, emotion, accomplishment and artistic accomplishment of concentration, different performance of different style of artistic beauty, such as a cursive style number, coherent whole, independent and traction permeate every word, not only shows the elegant calligraphy natural and unrestrained, independence, flowing, lively rhythm beauty, and conveys the calligrapher's open-minded, liberal, clever mind and artistic pursuit.

\section{Rhythm Beauty}

The beauty of Chinese characters is through the point and line as the carrier, through aesthetic experience to express the formal beauty, expressiveness and intensity of calligraphy. It's not like painting, sculpture, with image representation, can use different style, different art style white stipple the nexus of contracts, composition of cloth and even the whole artistically to present a different rhythm beauty. Rhythmic beauty is an indispensable factor to fully express the aesthetic taste and aesthetic characteristics of Chinese characters. The characters of Chinese characters have a strong artistic quality, which can reflect the beauty of the dynamic rhythm and the beauty of natural image and the beauty of implied meaning through static points and lines. The oracle "rain" means drops of water 
falling from the clouds; "Step" means the shape of the foot that is walking; The seal script "chuan" indicates that the dynamic of the liquid downward flow vividly expresses the beauty of Chinese characters' meaning and rhythm, as shown in Fig.6. The simple, clear, dynamic point and line of the Chinese characters are very vivid and distinctive in their relationship with nature, which is exactly what the art design needs.
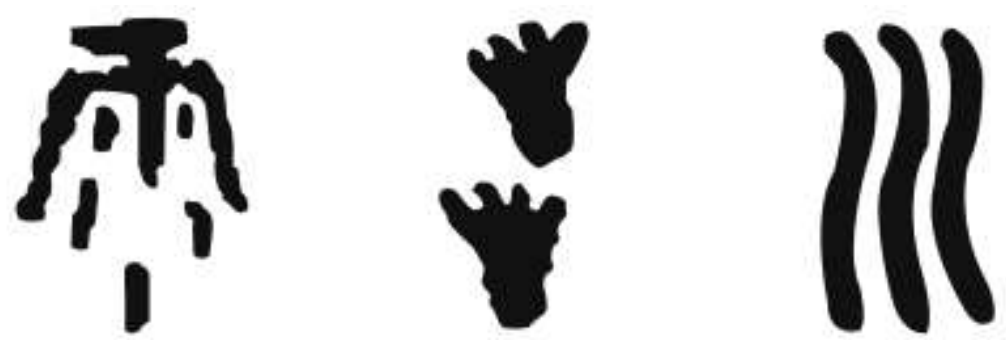

Figure 6. Oracle-bone: "rain", Bronze inscriptions: "step", Seal character "river"

The characters of Chinese characters have been extremely rhythmic and beautiful. On the one hand, for thousands of years, calligraphers have created a variety of beautiful and beautiful works of rhythmic beauty. For example, before the beginning of the tang dynasty, "the book is very thin and hard to make the gods", and after the middle tang, "rich and powerful" is the pursuit of beauty. On the other hand, the stroke of Chinese characters also has a strong sense of rhythm. Points, horizontal, vertical, left, si, folding, bending, hook has a subtle rhythm between square, contains the size, length, soft, light and heavy, the ups and downs, echo contradictions, such as font and put, fast, slow, stop and, according to the present endless pen and ink interest, achieve a silent sound, static wins the beauty of rhythm. As Japan's tanaka said, "the pen and ink are not painted, they are not necessarily words, they are only the brush with the ink to show a graceful trajectory." [7] as si said, the rhythmic beauty contained in Chinese characters has a very important enlightenment effect on art design.

\section{The Application of Artistic and Rhythmic Beauty in Design}

The beauty of Chinese characters is through the point and line as the carrier, through aesthetic experience to express the formal beauty, expressiveness and intensity of calligraphy. It's not like painting, sculpture, with image representation, can use different style, different art style white stipple the nexus of contracts, composition of cloth and even the whole artistically to present a different rhythm beauty. Rhythmic beauty is an indispensable factor to fully express the aesthetic taste and aesthetic characteristics of Chinese characters. The characters of Chinese characters have a strong artistic quality, which can reflect the beauty of the dynamic rhythm and the beauty of natural image and the beauty of implied meaning through static points and lines. The oracle "rain" means drops of water falling from the clouds; "Step" means the shape of the foot that is walking; The seal script "chuan" indicates that the dynamic of the liquid downward flow vividly expresses the beauty of Chinese characters' meaning and rhythm, as shown in Fig.7. The simple, clear, dynamic point and line of the Chinese characters are very vivid and distinctive in their relationship with nature, which is exactly what the art design needs. 


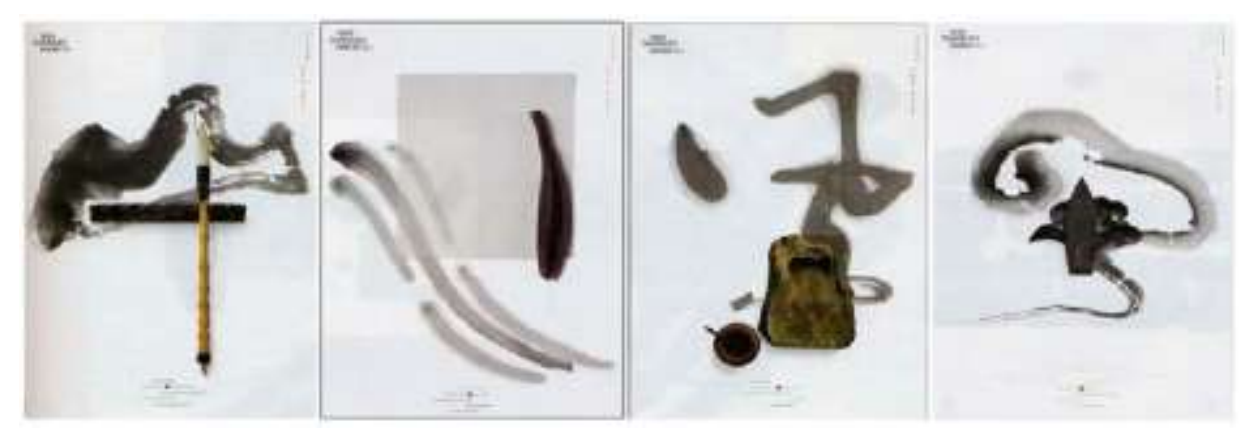

Figure 7. "Chinese characters" series of posters

\section{The Design and Emotional Beauty of Chinese Characters}

\section{Design and Emotional Beauty}

The design of Chinese characters refers to the use of stroke, structure and image features to transform Chinese characters into vivid visual shapes. The Chinese characters in the form of points and lines are the basic principles for the design requirements to convey the design intention directly and quickly. Chinese characters design personalized and easy to spread of Chinese characters itself, the characteristics of Chinese character graphics over a previous traditional routine monotonous type of writing form, into the characteristics of modern decoration art, combining the feature of own rationality and vitality, are more likely to stimulate the audience's visual perception alertness, which makes design [8]. As a kind of pure art form, Chinese characters can express their thoughts and emotions in a sincere way, and show their aesthetic pursuit in an honest and simple way, so that the works can express the beauty of emotion in a simple and naive way. If the calligraphy works of different styles or the implicit or unrestrained, or the elegant and gentle, or the precipitous release all reveals the different design and the emotional beauty. As Chen Yi of the Yuan Dynasty once said, "we all have scores. Happiness is qi and the word, anger is bold and word risk, sad is qi yu, the word is restrained, the music is flat and the word li. Love is light, but the word is also shallow and profound, the change is infinite. [9]

\section{The Application Of Design and Emotional Beauty in Design}

In terms of design and emotional beauty, Chinese characters can be used in a wide range of elements, such as the pendants in calligraphy strokes, the inverted stamen, the waves, the drooping, the flying white, the dry pen, etc. In the design, ingenious use of characteristics of Chinese character strokes, cutting through art gimmick to add and subtract, exaggerated deformation can be obtained unexpected, interesting, emotion, profound in artistic effect. Through the design of Chinese characters, it conveys the value orientation, aesthetic appeal and thought emotion in the form of literal meaning, painting, painting and painting. For example, the "Fu" paper in traditional paper-cut contains 12 zodiac forms, which contains the good wishes of "year after year". The longevity star, crane, longevity peach and pine tree symbolize longevity and longevity, as shown in Fig.8. 

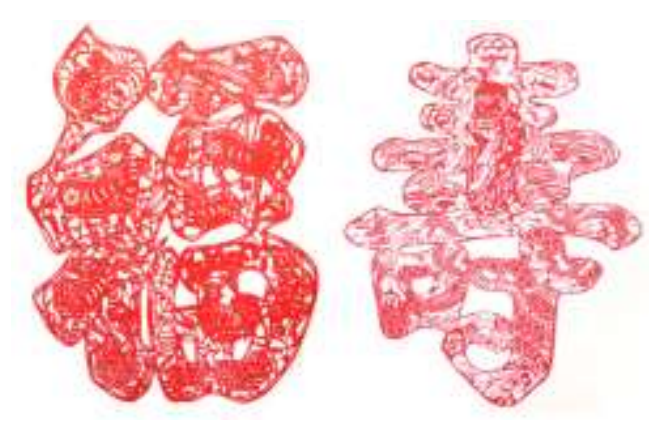

Figure 8. Folk paper-cut: "blessing", "Shou"

In the design, Chinese characters as a design element, its design and emotional beauty to the audience through different font and form different psychological feelings, the emotional theory in the works contains the merge convey accurate and effective design goals are met. The research on the design and emotional beauty of Chinese characters is beneficial to the application of traditional culture as the design element to the design. Such as Chinese handwriting and flexibility, graceful form, have Gentle and elegant. neatly and lively beauty, regular script font specification, statutes, rigorous, easy to identify, with "elegant" aesthetic emotion, founder of song typeface stiffness, handwriting cohesion, echo of point, line, give a person the sense kind, the bold solemn marked, neat and balanced symmetrical structure, make the person has contracted fashion beauty of emotion. Visible, different forms of Chinese characters convey emotion beauty in design is different, some elegant, some lively, some elegant, some beautiful, reasonable utilization of the emotional beauty of Chinese characters to make works convey different emotions, reduce the distance with the target audience, produce emotional resonance. Jin Daiqing design "at ease" patterned paper posters, through the small tadpoles free "play", straw sandals traveled the side of the "line", eat mulberry leaves no rules no beam "eat", water lily repose a limpid "sleep", make the theme of the comfortable playing ", line, eat, sleep "figure, form one integrated mass. Chinese calligraphy to frustration, traditional ink painting unique dry wet shade, modelling is wonderful, the butterflies, make whole works in a contemporary, traditional performance both the paper and the harmony of man and nature, and shows the heart and the natural harmony. Show a carefree spirit of life, and tell the beauty of emotions that can't be said, as shown in Fig.9.
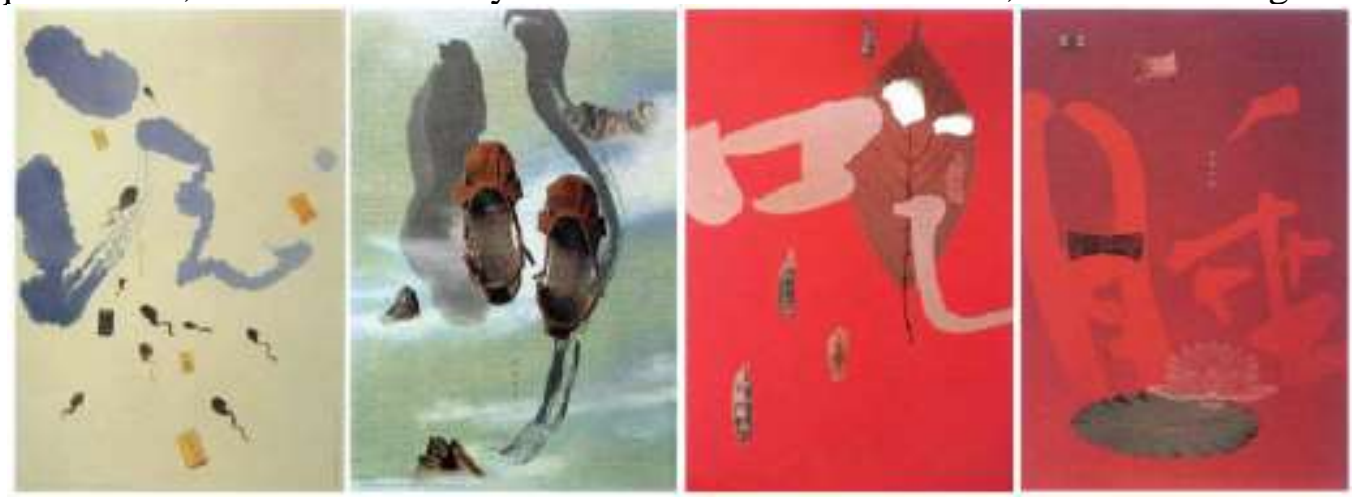

Figure 9. Folk paper-cut: "blessing", "Shou"

\section{Conclusion}

In the field of design, more consideration to the aesthetic features of Chinese characters and the relationship between the art and design in expression form, to gain a strong visual impact, so as to realize works to express design intent and objectives. With the development of economy, science and 
technology and culture, art design has entered a new period of rapid development. The design and application of Chinese characters have a deeper understanding and experience, and the demand for the aesthetic characteristics of Chinese characters is getting higher and higher. Exploring Chinese traditional culture, deeply research and application of inclusion of the aesthetic features of art and design development prospect and play a better the ethnic flavor of local design has very important value and significance.

However, in the design, it is necessary to avoid mistakes in the creation, design and aesthetic characteristics of Chinese characters. The effective principles and methods are: first, to grasp the influence of Chinese characters on design in the way of thinking. The image thinking, main body thinking and combination thinking of Chinese characters can effectively stimulate design thinking. Chinese elements should pay attention to in the design of "means" and "lenovo" national aesthetic psychology and creative thinking method of Chinese characters of the sound, form, meaning and the design of organic integration, the work of design goal and the audience's psychology to get good communication. Second, grasp the influence of Chinese characters on the design. The expression of Chinese characters' re - shape and heavy meaning has a beneficial effect on the design method. According to the idea, starting from the iconicity, artistic quality and design of Chinese characters, the key to grasp the relationship of form and meaning, Chinese characters, such as Chinese characters with freehand brushwork, like composite, a cane melon and in the form of word expression of fictional reality, stroke by stroke deformation trade-off in the design, form of subversion, isomorphic isomorphism, abnormity, decomposition reconstruction, to knot words, such as element fusion method is applied to design of Chinese characters in ably and rebirth.

Third, grasp the influence of Chinese characters on design. The influence of Chinese character poetry on design is mainly reflected in the artistic conception and emotional connotation of the works. In the design, through the Chinese characters of the structure beauty, the rhythm of beauty, the emotional beauty, the use of imagery, symbolization of the artistic techniques to achieve the poetic visual effect. Make work has the poetic beauty of attitude of life, showing both the image, the symbol of "real", outside is like the "empty", so as to achieve the "real" physical function to the spirit of "virtual" aesthetic sublimation. It can be seen that only by following these principles and methods, can Chinese characters express rich content in a concise, novel and effective form in artistic design to achieve the expected goal. "In order to better use the traditional design elements of the Chinese character, to make it more artistic charm, to reflect the more intense national meaning and Oriental charm".[10]

\section{References}

[1] QU Mei. Visual Deconstruction of "Imagery Spirit" in ChineseCharacters Poster Design[J].Packaging Engineering,2014,35

[2] XU Zi-hong.Book Translation[M].Guiyang: Guizhou PeoplePress, 2009.

[3] WANG Bo-min.The Theory of Calligraphy Integration[M].Shijiazhuang : Hebei Fine Arts Publishing House, 2002.

[4] ZONG Bai-hua.Strolling in Aesthetics[M].Shanghai: Shanghai People Publishing House,1981.

[5] LIU Juan.New Century Design Chinese Characters of Hybridity and Change[J].Seeker,2012,31 (11): $120-121$.

[6] LI Ze-hou.Aesthetic Trilogy[M].Tianjin: Tianjin Academy of Social Sciences Press,2011.

[7] WANG Xu.The Design of the Jindaiqiang Graphic Designe[M].Beijing: China Youth Press, 1999.

[8] CHEN Shan-shan. On the Effective Application of Chinese Characters Culture on Modern Graphic Design[J].Packaging Engineering,2013,34 (16): 119-122.

[9] The Collation of Ancient Books Research Room of East China Normal University.The Calligraphy 
Selected Essays of Successive Dynasties[M].Shanghai : Shanghai Fine Arts Publishing House, 1979.

[10] YE Ming-hui.Interpretation of "the Movement of Character " : an Overview on the Wave of Contemporary Chinese Typography[J].Journal of Shenzhen University,2013,30（5): 170—175.

Author introduction: Sun Hong-yang (1975 -), Male, Jiangxi Xiushui, associate professor, master of fine arts education, supervisor of postgraduate, the Art Institute of East China University of Technology, Visual Communication Department, South Korea Chonbuk National University studying for a PhD, mainly engaged in the study of painting theory and art design. 